\title{
QUASILINEAR ELLIPTIC EQUATIONS \\ WITH SINGULAR POTENTIALS \\ AND BOUNDED DISCONTINUOUS NONLINEARITIES
}

\author{
Anran Li - Hongrui CAI - JiabaO SU
}

ABStRaCt. In this paper we study the quasilinear equation

(P) $\left\{\begin{array}{l}-\operatorname{div}\left(|\nabla u|^{p-2} \nabla u\right)+V(|x|)|u|^{p-2} u=Q(|x|) f(u), \quad x \in \mathbb{R}^{N}, \\ u(x) \rightarrow 0, \quad|x| \rightarrow \infty .\end{array}\right.$

with singular radial potentials $V, Q$ and bounded measurable function $f$. The approaches used here are based on a compact embedding from the space $W_{r}^{1, p}\left(\mathbb{R}^{N} ; V\right)$ into $L^{1}\left(\mathbb{R}^{N} ; Q\right)$ and a new multiple critical point theorem for locally Lipschitz continuous functionals.

\section{Introduction}

In this paper we are concerned with the quasilinear elliptic equation on $\mathbb{R}^{N}$

$(\mathrm{P})$

$$
\left\{\begin{array}{l}
-\operatorname{div}\left(|\nabla u|^{p-2} \nabla u\right)+V(|x|)|u|^{p-2} u=Q(|x|) f(u), \quad x \in \mathbb{R}^{N}, \\
u(x) \rightarrow 0, \quad|x| \rightarrow \infty
\end{array}\right.
$$

2010 Mathematics Subject Classification. 35J10, 35J65, 58E05.

Key words and phrases. Quasilinear elliptic equation, Sobolev embedding, non-smooth critical point theorem, bounded discontinuous nonlinearity.

This work was supported by NSFC11271264, NSFC11171204 and PHR201106118. 
where $1<p<N, N \geq 3$ and $V, Q:(0, \infty) \mapsto(0, \infty)$ are two continuous functions satisfying the assumptions

$(\mathrm{V})$ there exist real numbers $a$ and $a_{0}$ such that

$$
\liminf _{r \rightarrow \infty} \frac{V(r)}{r^{a}}>0, \quad \liminf _{r \rightarrow 0} \frac{V(r)}{r^{a_{0}}}>0 .
$$

(Q) there exist real numbers $b$ and $b_{0}$ such that

$$
\limsup _{r \rightarrow \infty} \frac{Q(r)}{r^{b}}<\infty, \quad \limsup _{r \rightarrow 0} \frac{Q(r)}{r^{b_{0}}}<\infty
$$

The nonlinear function $f$ satisfies the following conditions:

$\left(\mathrm{f}_{1}\right) f: \mathbb{R} \rightarrow \mathbb{R}$ is a measurable function;

$\left(\mathrm{f}_{2}\right)$ there is $C_{1}>0$ such that $|f(u)| \leq C_{1}$, for $u \in \mathbb{R}$;

$\left(\mathrm{f}_{3}\right)$ there is $C_{2}>0$ such that $F(u)=\int_{0}^{u} f(t) d t \geq C_{2}|u|$, for $u \in \mathbb{R}$;

$\left(\mathrm{f}_{4}\right) f(-u)=-f(u), u \in \mathbb{R}$.

The function $f(u)=\operatorname{sgn} u$ satisfies the conditions $\left(\mathrm{f}_{1}\right)-\left(\mathrm{f}_{4}\right)$. By $\left(\mathrm{f}_{1}\right)$ and $\left(\mathrm{f}_{2}\right)$, $F(u)=\int_{0}^{u} f(t) d t$ is locally Lipschitz continuous, and it may not be differentiable since there is no continuous function satisfying simultaneously $\left(\mathrm{f}_{3}\right)$ and $f(0)=0$. It follows from $\left(\mathrm{f}_{4}\right)$ that $(\mathrm{P})$ has a trivial solution $u=0$, we are interested in multiple nontrivial solutions related to $(\mathrm{P})$ in some sense due to the symmetry of nonlinearity $f$.

We will apply the variational methods to $(\mathrm{P})$. To this end we establish the variational framework associated to $(\mathrm{P})$. Denote by $D_{r}^{1, p}\left(\mathbb{R}^{N}\right)$ the completion of radial functions $C_{0, r}^{\infty}\left(\mathbb{R}^{N}\right)$ under the norm

$$
\|\nabla u\|_{L^{p}}=\left(\int_{\mathbb{R}^{N}}|\nabla u|^{p} d x\right)^{1 / p} .
$$

Define for $p>1$

$$
\begin{aligned}
& L^{p}\left(\mathbb{R}^{N} ; V\right):=\left\{u: \mathbb{R}^{N} \mapsto \mathbb{R} \mid u\right. \text { is Lebesgue measurable, } \\
& \left.\qquad \int_{\mathbb{R}^{N}} V(|x|)|u|^{p} d x<\infty\right\} .
\end{aligned}
$$

Then define

$$
W_{r}^{1, p}\left(\mathbb{R}^{N} ; V\right):=D_{r}^{1, p}\left(\mathbb{R}^{N}\right) \cap L^{p}\left(\mathbb{R}^{N} ; V\right),
$$

which is a Banach space (see [1], [15]) equipped with the norm

$$
\|u\|_{W_{r}^{1, p}\left(\mathbb{R}^{N} ; V\right)}=\left(\int_{\mathbb{R}^{N}}\left(|\nabla u|^{p}+V(|x|)|u|^{p}\right) d x\right)^{1 / p} .
$$

Define

$L^{1}\left(\mathbb{R}^{N} ; Q\right):=\left\{u: \mathbb{R}^{N} \mapsto \mathbb{R} \mid u\right.$ is Lebesgue measurable, $\left.\int_{\mathbb{R}^{N}} Q(|x|)|u| d x<\infty\right\}$. 
In [12], the following compact embedding theorem has been established.

Theorem 1.1 (Theorem 2.1 in [12]). Assume (V) and (Q) with

$$
\begin{gathered}
b_{0}>\min \left\{\frac{N-p}{p}-N, \frac{p(N-1)+a_{0}(p-1)}{p^{2}}-N\right\}, \\
b<\max \left\{\frac{N-p}{p}-N, \frac{p(N-1)+a(p-1)}{p^{2}}-N\right\} .
\end{gathered}
$$

Then the embedding $W_{r}^{1, p}\left(\mathbb{R}^{N} ; V\right) \hookrightarrow L^{1}\left(\mathbb{R}^{N} ; Q\right)$ is compact.

By the above compact embedding from $W_{r}^{1, p}\left(\mathbb{R}^{N} ; V\right)$ into $L^{1}\left(\mathbb{R}^{N} ; Q\right)$, the functional related to $(\mathrm{P})$

$$
\Phi(u)=\frac{1}{p} \int_{\mathbb{R}^{N}}\left(|\nabla u|^{p}+V(|x|)|u|^{p}\right) d x-\int_{\mathbb{R}^{N}} Q(|x|) F(u) d x
$$

is well defined on $W_{r}^{1, p}\left(\mathbb{R}^{N} ; V\right)$ and is locally Lipschitz continuous on $W_{r}^{1, p}\left(\mathbb{R}^{N} ; V\right)$ when $\left(f_{1}\right)$ and $\left(f_{2}\right)$ are assumed. In addition, $\Phi$ is an even functional when $\left(f_{4}\right)$ is assumed. We will apply the nonsmooth critical point theory built in [2] to study (P) by looking for critical points of $\Phi$ on $W_{r}^{1, p}\left(\mathbb{R}^{N} ; V\right)$.

The main result in this paper reads as follows.

THEOREM 1.2. Assume (V) and (Q) with

$$
\begin{gathered}
b_{0}>\min \left\{\frac{N-p}{p}-N, \frac{p(N-1)+a_{0}(p-1)}{p^{2}}-N\right\}, \\
b<\max \left\{\frac{N-p}{p}-N, \frac{p(N-1)+a(p-1)}{p^{2}}-N\right\} .
\end{gathered}
$$

If $f$ satisfies $\left(\mathrm{f}_{1}\right)-\left(\mathrm{f}_{4}\right)$, then the functional $\Phi$ related to $(\mathrm{P})$ has infinitely many critical points $u_{k} \in W_{r}^{1, p}\left(\mathbb{R}^{N} ; V\right)$ with negative energy $\Phi\left(u_{k}\right):=c_{k} \rightarrow 0$ as $k \rightarrow \infty$.

We give some comments, explanations and comparisons. We have pointed out that there is no continuous function $f$ that satisfies the assumptions $\left(\mathrm{f}_{1}\right)-\left(\mathrm{f}_{4}\right)$. Therefore the problem $(\mathrm{P})$ is a kind of nonlinear partial differential equation with discontinuous nonlinearities. In the past decades many efforts have been devoted in extending the theory of nonlinear partial differential equations (PDE) to PDE with discontinuous nonlinearities (DPDE). We refer the readers to the pioneering works [2]-[4] and the references therein for some historic developments and the explanations in view of physical and mathematical aspects. The abstract methods for dealing with DPDE have been developed. In [2], the author established variational methods for non-differentiable functional by using the generalized gradients for locally Lipschitz continuous functions on Banach space introduced by Clarke [5]. In [2] the concept of critical points, the Palais-Smale condition and the deformation lemma to suitable classes of non-differentiable functionals 
were extended, and various minimax theorems also had been extended. Since then, other kinds of non-smooth critical point theory were established, see the references [6], and [9] and the references therein. In [6], the author studied the existence of solution of some quasilinear equations via non-smooth Morse Theory.

In the literature most known results by applying non-smooth critical point theory were concerned with nonlinear elliptic equations on a bounded domain $\Omega$ of $\mathbb{R}^{N}$. Since the classical Sobolev embedding theorems worked well, in general the nonlinearities have a subcritical growth and may be classified as sublinear, asymptotically linear, or superlinear. See [2], [7], [9].

It is well known that the Sobolev compact embedding theorems play a crucial role in dealing with elliptic problems via variational methods ([10], [17]). When one deals with the semilinear elliptic problem on a bounded domain $\Omega$ of $\mathbb{R}^{N}$, the classical Sobolev embedding from $H^{1}(\Omega)$ into $L^{q}(\Omega)$ is compact for all $1 \leq$ $q<2^{*}:=2 N /(N-2), N \geq 3$. The case $1 \leq q<2$ is from a fact of continuous embedding $L^{q}(\Omega) \subset L^{s}(\Omega)$ for $1 \leq s \leq q$. On the whole space $\mathbb{R}^{N}$, the embedding $H^{1}\left(\mathbb{R}^{N}\right) \hookrightarrow L^{q}\left(\mathbb{R}^{N}\right)$ is only continuous for $q \in\left[2,2^{*}\right]$ and is not compact for any $q$. Restricted to the radial case, $H_{r}^{1}\left(\mathbb{R}^{N}\right) \hookrightarrow L^{q}\left(\mathbb{R}^{N}\right)$ are compact for all $q \in\left(2,2^{*}\right.$ ) (see [11], [17]), and it is not true for $q=2$ and $q=2^{*}$. When radial potentials are involved, the compactness of the embedding may be valid for a wider range of $q$. In [14], [15], the authors developed techniques and ideas in establishing weighted Sobolev embedding from $W_{r}^{1, p}\left(\mathbb{R}^{N} ; V\right)$ into $L^{q}\left(\mathbb{R}^{N} ; Q\right)$ with singular radial potentials $V$ and $Q$ for $q \geq p$ that provided a basic tool in studying $(\mathrm{P})$ with super-p-linear nonlinearity. In [13] the authors further explored the effects of the potentials and extend with $1<q<p$ and then to study $(\mathrm{P})$ with sub-p-linear nonlinearity. In [12] the compact embedding $W_{r}^{1, p}\left(\mathbb{R}^{N} ; V\right) \hookrightarrow L^{1}\left(\mathbb{R}^{N} ; Q\right)$ (Theorem 1.1) was established and then was applied to the solvability of the quasilinear problem $(\mathrm{P})$ with a bounded continuous nonlinearity $f$. Since there is no relation between $L^{q}\left(\mathbb{R}^{N} ; Q\right)$ and $L^{s}\left(\mathbb{R}^{N} ; Q\right)$ for any $q$ and $s$, the embedding from $W_{r}^{1, p}\left(\mathbb{R}^{N} ; V\right)$ into $L^{q}\left(\mathbb{R}^{N} ; Q\right)$ are independent from each other for $q \geq p, 1<q<p$ and $q=1$.

The novelty of this paper are two-fold. One is that we consider the quasilinear elliptic equations $(\mathrm{P})$ on the whole space $\mathbb{R}^{N}$ with a bounded discontinuous nonlinearity and the variational framework of $(\mathrm{P})$ is based only on the compact embedding Theorem 1.1. Another one is that we want to find multiple critical points of $\Phi$ via nonsmooth critical point theory. There is not any an abstract tool in the literature which can be applied directly to Theorem 1.2. We will establish a new multiple critical point theorem for non-smooth functional which extends one theorem in [16] for smooth functional. The result of Theorem 1.2 is completely new and completes the statement in the end of [12]. We point out that this type of result is even new for the semilinear elliptic boundary value 
problem on bounded domains with discontinuous nonlinearities. In this case $\left(\mathrm{f}_{3}\right)$ may be replaced with other conditions and no weighted functions are involved. See remarks below in the end of this paper.

The paper is organized as follows. In Section 2, we establish a multiple critical point theorem for locally Lipschitz continuous functionals. We will use the variational framework for non-differentiable functionals built in [2]. In Section 3, we give the proof of Theorems 1.2 and give further remarks.

\section{A nonsmooth critical point theorem}

In this section we establish an abstract critical point theorem for locally Lipchitz functionals. We follow up with the variational framework built in [2] for the non-differentiable functionals.

Let $X$ be a real Banach space and $X^{*}$ be its dual space. For $u \in X, u^{*} \in X^{*}$, $\left\langle u^{*}, u\right\rangle$ denotes the duality between $X^{*}$ and $X$. Let $\Phi: X \rightarrow \mathbb{R}$ be a locally Lipschitz continuous functional, that is, for each $u \in X$, there is a neighbuorhood $\mathbb{N}$ of $u$ and a constant $K$ depending on $\mathbb{N}$ such that

$$
|\Phi(y)-\Phi(z)| \leq K\|y-z\| \quad \text { for all } y, z \in \mathbb{N} \text {. }
$$

For each $v \in X$, the generalized directed derivative $\Phi^{0}(u ; v)$ of $\Phi$ at $u \in X$ in the direction $v$ is defined as

$$
\Phi^{0}(u ; v)=\limsup _{h \rightarrow 0, \lambda \downarrow 0} \frac{1}{\lambda}[\Phi(u+h+\lambda v)-\Phi(u+h)] .
$$

The generalized directed derivative $\Phi^{0}(u ; \cdot)$ enjoys some basic properties [5]: for each $u \in X$, the function $v \rightarrow \Phi^{0}(u ; v)$ is continuous on $v$ and satisfies $\left|\Phi^{0}(u ; v)\right| \leq K\|v\|$, and furthermore, it is subadditive, positively homogenous, and then is convex. We refer to [5] for these facts.

Definition 2.1 (Clarke [5]). Let $\Phi: X \rightarrow \mathbb{R}$ be a locally Lipschitz continuous functional. The generalized gradient of $\Phi$ at $u \in X$, denoted by $\partial \Phi(u)$, is defined to be the sub-differential of the convex function $\Phi^{0}(u ; v)$ at $v=0$ :

$$
\partial \Phi(u):=\left\{\omega \in X^{*} \mid\langle\omega, v\rangle \leq \Phi^{0}(u ; v) \text { for all } v \in X\right\}
$$

We refer the readers to [2] for some general information about the generalized gradient.

We need the following concepts.

Definition $2.2([2])$. Let $\Phi: X \rightarrow \mathbb{R}$ be a locally Lipschitz functional. We say that $u_{0} \in X$ is a critical point of $\Phi$ if $0 \in \partial \Phi\left(u_{0}\right)$. 
Definition $2.3([2])$. Let $\Phi: X \rightarrow \mathbb{R}$ be a locally Lipschitz functional. We say that $\Phi$ satisfies the Palais-Smale condition if any sequence $\left\{u_{n}\right\} \subset X$ along which $\Phi\left(u_{n}\right)$ is bounded and

$$
\lambda\left(u_{n}\right)=\min _{\omega \in \partial \Phi\left(u_{n}\right)}\|\omega\|_{X^{*}} \rightarrow 0
$$

possesses a convergent subsequence.

For $c \in \mathbb{R}$, we denote

$$
\Phi^{c}=\{u \in X \mid \Phi(u) \leq c\} ; \quad \mathbb{K}_{c}=\{u \in X \mid 0 \in \partial \Phi(u), \Phi(u)=c\} .
$$

We note here that $\mathbb{K}_{c}$ is compact if $\Phi$ satisfies the Palais-Smale condition. See Lemma 3.1 in [2]. The following deformation lemma for locally Lipschitz functional was proved in [2].

Lemma 2.4 ([2]). Suppose that $X$ is a reflexive Banach space, and $\Phi$ is a locally Lipschitz functional and satisfies the Palais-Smale condition. If $c$ is a real number and $\mathbb{N}$ is any a neighbourhood of the set $\mathbb{K}_{c}$, then for any $\varepsilon_{0}>0$, there exists $\varepsilon \in\left(0, \varepsilon_{0}\right)$ and a homomorphism $\eta: X \rightarrow X$ such that

(a) $\eta(u)=u$ for $u \notin \Phi^{c+\varepsilon_{0}} \backslash \Phi^{c-\varepsilon_{0}}$;

(b) $\eta\left(\Phi^{c+\varepsilon} \backslash \mathbb{N}\right) \subset \Phi^{c-\varepsilon}$;

(c) if $\mathbb{K}_{c}=\emptyset$, then $\eta\left(\Phi^{c+\varepsilon}\right) \subset \Phi^{c-\varepsilon}$;

(d) if $\Phi$ is even, then the homomorphism $\eta: X \rightarrow X$ is an odd mapping.

Now we apply Lemma 2.4 to establish the following abstract critical point theorem for locally Lipschitz functionals. It can be regarded as the non-smooth version of an abstract critical point theorem in Wang [16] (see Lemma 2.4 in [16]) for smooth functionals. For readers convenience we sketch a proof along with the arguments of [16].

Theorem 2.5. Let $X$ be a reflexive Banach space and $\Phi: X \rightarrow \mathbb{R}$ be a locally Lipschitz functional. Assume that $\Phi$ satisfies Palais-Smale condition and is even, bounded from below, $\Phi(0)=0$. If for any $k \in \mathbb{N}$, there exists a $k$ dimensional subspaces $X_{k}$ and $\rho_{k}>0$, such that

$$
\sup _{u \in X_{k} \cap S_{\rho_{k}}} \Phi(u)<0,
$$

where $S_{\rho}=\{u \in X \mid\|u\|=\rho\}$, then $\Phi$ has a sequence of critical values $c_{k}<0$ satisfying $c_{k} \rightarrow 0$ as $k \rightarrow \infty$.

Proof. Let $\Sigma$ be the class of closed symmetric subsets of $X \backslash\{0\}$. For $A \in \Sigma$, we denote by $\gamma(A)$ the genus of $A$ (see [10]) defined as

$$
\gamma(A):=\min \left\{k \in \mathbb{N} \mid \text { there exists an odd map } \phi \in C\left(A, \mathbb{R}^{k} \backslash\{0\}\right)\right\} .
$$


One can refer to Proposition 7.5 in [10] for the properties of the genus $\gamma$. For each $k \in \mathbb{N}$, define the minimax value as

$$
c_{k}:=\inf _{A \in \Sigma, \gamma(A) \geq k} \sup _{u \in A} \Phi(u) .
$$

By the monotonicity property of the genus we have $c_{k} \leq c_{k+1}$ for all $k \in \mathbb{N}$. A standard argument applying Lemma 2.4 shows that all $c_{k}$ are critical values of $\Phi$. Assume that for some $k, c_{k}$ is not a critical value of $\Phi$. Then by Lemma 2.4(c), there exists $\varepsilon>0$ small and an odd homomorphism $\eta: X \rightarrow X$ such that $\eta\left(\Phi^{c_{k}+\varepsilon}\right) \subset \Phi^{c_{k}-\varepsilon}$.

Take $A \in \Sigma$ such that $\gamma(A) \geq k$ and $\sup _{u \in A} \Phi(u) \leq c_{k}+\varepsilon$, then $A \subset \Phi^{c_{k}+\varepsilon}$. Set $\widetilde{A}=\eta(A)$. Then $\widetilde{A} \in \Sigma$ and by the mapping property of the genus, we have

$$
\gamma(\widetilde{A})=\gamma(\eta(A)) \geq \gamma(A) \geq k .
$$

Thus from $\widetilde{A}=\eta(A) \subset \Phi^{c_{k}-\varepsilon}$, we deduce that

$$
c_{k} \leq \sup _{u \in A} \Phi(\eta(u)) \leq c_{k}-\varepsilon
$$

This is a contradiction.

Since $\gamma\left(X_{k} \cap S_{\rho_{k}}\right)=k([10])$, we have by (2.1) and (2.2) that $c_{k}<0$, for all $k \in \mathbb{N}$.

We now show that $c_{k} \rightarrow 0$, as $k \rightarrow \infty$. Assume that $c_{k} \rightarrow c<0, k \rightarrow \infty$. Since $\Phi$ satisfies the Palais-Smale condition, $\mathbb{K}_{c}$ is compact. Moreover, $\mathbb{K}_{c}$ is symmetric and $0 \notin \mathbb{K}_{c}$. Thus $\mathbb{K}_{c} \in \Sigma, \gamma\left(\mathbb{K}_{c}\right):=\ell<\infty$.

By the properties of genus, there is a closed neighbourhood $U$ of $\mathbb{K}_{c}$ such that $\mathbb{K}_{c}$ is a subset of the interior $U^{\circ}$ of $U$ and $\gamma(U)=\ell$.

By Lemma 2.4, there is $\varepsilon>0$ with $c+\varepsilon<0$ and an odd homeomorphism $\eta: X \rightarrow X$ such that $\eta\left(\Phi^{c+\varepsilon} \backslash U^{\circ}\right) \subset \Phi^{c-\varepsilon}$. Therefore

$$
\gamma\left(\Phi^{c+\varepsilon}\right) \leq \gamma\left(\Phi^{c+\varepsilon} \backslash U^{\circ}\right)+\gamma(U) \leq \gamma\left(\eta\left(\Phi^{c+\varepsilon} \backslash U^{\circ}\right)\right)+\ell \leq \gamma\left(\Phi^{c-\varepsilon}\right)+\ell .
$$

Taking $k \in \mathbb{N}$ large such that $c-\varepsilon<c_{k} \leq c$. Then $\gamma\left(\Phi^{c-\varepsilon}\right) \leq k$. Otherwise we would get $\gamma\left(\Phi^{c-\varepsilon}\right) \geq k+1$ which implies a contradiction $c_{k+1} \leq c-\varepsilon<c_{k}$. Therefore $\gamma\left(\Phi^{c+\varepsilon}\right) \leq k+\ell<\infty$. But, it is obviously that $\gamma\left(\Phi^{c+\varepsilon}\right)=\infty$. We have a contradiction and thus $c=0$.

We finally cite the following abstract result from [2] for proving our existence theorem.

Proposition 2.6 (Theorem 2.2 in [2]). Let $X$ and $Y$ be two Banach spaces. Assume that $X$ is reflexive, the embedding $X \hookrightarrow Y$ is continuous and $X$ is dense in $Y$. Let $\widetilde{G}$ be a locally Lipschitz continuous functional in $Y$, and $G=\left.\widetilde{G}\right|_{X}$, then

$$
\partial G(u) \subset \partial \widetilde{G}(u), \quad u \in X
$$




\section{Proof of Theorem 1.2}

In this section we give the proof of Theorem 1.2 by applying Theorem 2.5 . We first investigate that under the assumptions of Theorem 1.2, the variational framework of $(\mathrm{P})$ fits in with the variational framework in Section 2.

By the embedding Theorem 1.1, the functional

$$
\Phi(u)=\frac{1}{p} \int_{\mathbb{R}^{N}}\left(|\nabla u|^{p}+V(|x|)|u|^{p}\right) d x-\int_{\mathbb{R}^{N}} Q(|x|) F(u) d x, \quad u \in W_{r}^{1, p}\left(\mathbb{R}^{N} ; V\right)
$$

is well defined. Set the functional

$$
\Psi(u)=\frac{1}{p} \int_{\mathbb{R}^{N}}|\nabla u|^{p}+V(|x|)|u|^{p} d x, \quad u \in W_{r}^{1, p}\left(\mathbb{R}^{N} ; V\right) .
$$

Then $\Psi$ is of class $C^{1}$ on $W_{r}^{1, p}\left(\mathbb{R}^{N} ; V\right)$, and $\Psi^{\prime}$ possesses the $\left(\mathrm{S}^{+}\right)$property on $W_{r}^{1, p}\left(\mathbb{R}^{N} ; V\right)$ (see [8], [13]) in the sense that for any sequence $\left\{u_{n}\right\} \subset W_{r}^{1, p}\left(\mathbb{R}^{N} ; V\right)$, if

$$
u_{n} \rightarrow u \quad \text { weakly in } W_{r}^{1, p}\left(\mathbb{R}^{N} ; V\right) \text { and } \quad \limsup _{n \rightarrow \infty}\left\langle\Psi^{\prime}\left(u_{n}\right), u_{n}-u\right\rangle \leq 0,
$$

then

$$
u_{n} \rightarrow u \text { strongly in } W_{r}^{1, p}\left(\mathbb{R}^{N} ; V\right) .
$$

Set

$$
\widetilde{G}(u)=\int_{\mathbb{R}^{N}} Q(|x|) F(u) d x, \quad u \in L^{1}\left(\mathbb{R}^{N} ; Q\right) .
$$

By $\left(\mathrm{f}_{1}\right)$ and $\left(\mathrm{f}_{2}\right), \widetilde{G}$ is a uniformly Lipschitz continuous functional on $L^{1}\left(\mathbb{R}^{N} ; Q\right)$. In fact we have that

$$
|\widetilde{G}(u)-\widetilde{G}(v)| \leq \widehat{C}\|u-v\|_{L^{1}\left(\mathbb{R}^{N} ; Q\right)}, \quad \text { for } u, v \in L^{1}\left(\mathbb{R}^{N} ; Q\right) .
$$

Set $G=\left.\widetilde{G}\right|_{W_{r}^{1, p}\left(\mathbb{R}^{N} ; V\right)}$, then by Theorem 1.1 , we see that $G$ is a uniformly Lipschitz continuous functional on $W_{r}^{1, p}\left(\mathbb{R}^{N} ; V\right)$ :

$$
|G(u)-G(v)| \leq \widetilde{C}\|u-v\|_{W_{r}^{1, p}\left(\mathbb{R}^{N} ; V\right)}, \quad \text { for } u, v \in W_{r}^{1, p}\left(\mathbb{R}^{N} ; V\right) .
$$

The above constants $\widehat{C}$ and $\widetilde{C}$ are independent of the functions $u, v$.

Since $\Phi(u)=\Psi(u)-G(u)$, the functional $\Phi$ is a locally Lipschitz functional on $W_{r}^{1, p}\left(\mathbb{R}^{N} ; V\right)$.

We begin to prove Theorem 1.2. First of all, we prove that $\Phi$ is coercive and bounded from below.

Lemma 3.1. Assume that $f$ satisfies $\left(\mathrm{f}_{1}\right)$ and $\left(\mathrm{f}_{2}\right)$, then the functional $\Phi$ is coercive and bounded from below. 
Proof. For $u \in W_{r}^{1, p}\left(\mathbb{R}^{N} ; V\right)$, by $\left(\mathrm{f}_{2}\right)$, we have

$$
\begin{aligned}
\Phi(u) & =\frac{1}{p} \int_{\mathbb{R}^{N}}|\nabla u|^{p}+V(|x|)|u|^{p} d x-\int_{\mathbb{R}^{N}} Q(|x|) F(u) d x \\
& \geq \frac{1}{p}\|u\|_{W_{r}^{1, p}\left(\mathbb{R}^{N} ; V\right)}^{p}-C\|u\|_{L^{1}\left(\mathbb{R}^{N} ; Q\right)} \\
& \geq \frac{1}{p}\|u\|_{W_{r}^{1, p}\left(\mathbb{R}^{N} ; V\right)}^{p}-C\|u\|_{W_{r}^{1, p}\left(\mathbb{R}^{N} ; V\right)} .
\end{aligned}
$$

Since $p>1$, by $(3.1)$ we have

$$
\Phi(u) \rightarrow \infty \quad \text { as }\|u\|_{W_{r}^{1, p}\left(\mathbb{R}^{N} ; V\right)} \rightarrow \infty
$$

and $\Phi$ is bounded from below.

Next, we show that $\Phi$ satisfies the Palais-Smale condition. We will use Proposition 2.6. Set

$$
X=W_{r}^{1, p}\left(\mathbb{R}^{N} ; V\right), \quad Y={\overline{W_{r}^{1, p}\left(\mathbb{R}^{N} ; V\right)}}^{\|\cdot\|_{L^{1}\left(\mathbb{R}^{N} ; Q\right)}} .
$$

By Theorem 1.1, $X$ and $Y$ fit in with the conditions on spaces in Proposition 2.6 in the sense that $X$ is reflexive, the embedding $X \hookrightarrow Y$ is continuous (and compact), and $X$ is dense in $Y$. In the proof of the following lemma we use the notation $X$ instead of $W_{r}^{1, p}\left(\mathbb{R}^{N} ; V\right)$.

LEMma 3.2. Assume that $f$ satisfies $\left(\mathrm{f}_{1}\right)$ and $\left(\mathrm{f}_{2}\right)$, then the functional $\Phi$ satisfies the Palais-Smale condition.

Proof. Let $\left\{u_{n}\right\} \subset X$ be such that $\Phi\left(u_{n}\right)$ is bounded and

$$
\lambda\left(u_{n}\right)=\min _{\omega \in \partial \Phi\left(u_{n}\right) \subset X^{*}}\|\omega\|_{X^{*}} \rightarrow 0 \quad \text { as } n \rightarrow \infty .
$$

By the coercivity (3.2), $\left\{u_{n}\right\}$ is bounded in $X$. By the reflexivity of $X$ and the compactness of the embedding $X \hookrightarrow Y$, up to a subsequence if necessary, we may assume that there is $u \in X$ such that

$$
\begin{aligned}
& u_{n} \rightarrow u, \quad \text { weakly in } X, n \rightarrow \infty, \\
& u_{n} \rightarrow u, \quad \text { strongly in } Y \subset L^{1}\left(\mathbb{R}^{N} ; Q\right), n \rightarrow \infty .
\end{aligned}
$$

By the properties of the generalized gradient (see [2]), for each $n \in \mathbb{N}$, there exists $u_{n}^{*} \in \partial \Phi\left(u_{n}\right) \subset X^{*}$, such that

$$
\lambda\left(u_{n}\right)=\left\|u_{n}^{*}\right\|_{X^{*}},
$$

and there exists $v_{n}^{*} \in \partial G\left(u_{n}\right) \subset X^{*}$ such that

$(3.7)\left\langle u_{n}^{*}, v\right\rangle=\int_{\mathbb{R}^{N}}\left(\left|\nabla u_{n}\right|^{p-2} \nabla u_{n} \nabla v+V(|x|)\left|u_{n}\right|^{p-2} u_{n} v\right) d x-\left\langle v_{n}^{*}, v\right\rangle, \quad v \in X$. 
Taking $v=u_{n}-u$ in (3.7), then

$$
\begin{aligned}
& \left\langle\Psi^{\prime}\left(u_{n}\right), u_{n}-u\right\rangle \\
& \quad=\int_{\mathbb{R}^{N}}\left(\left|\nabla u_{n}\right|^{p-2} \nabla u_{n} \nabla\left(u_{n}-u\right)+V(|x|)\left|u_{n}\right|^{p-2} u_{n}\left(u_{n}-u\right)\right) d x \\
& \quad=\left\langle u_{n}^{*}, u_{n}-u\right\rangle+\left\langle v_{n}^{*}, u_{n}-u\right\rangle .
\end{aligned}
$$

By (3.3), (3.4) and (3.6), it follows that

$$
\left\langle u_{n}^{*}, u_{n}-u\right\rangle \rightarrow 0, \quad \text { as } n \rightarrow \infty .
$$

By Proposition 2.6, we see that $v_{n}^{*} \in \partial G\left(u_{n}\right) \subset \partial \widetilde{G}\left(u_{n}\right) \subset Y^{*}$. Since $\widetilde{G}$ is uniformly Lipschitz continuous on $Y$, we have by the properties of the generalized gradient (see [2]) that

$$
\left\|v_{n}^{*}\right\|_{Y^{*}} \leq \widehat{C}, \quad n \in \mathbb{N}
$$

It follows from (3.5) and (3.10) that

$$
\left|\left\langle v_{n}^{*}, u_{n}-u\right\rangle\right| \leq\left\|v_{n}^{*}\right\|_{Y^{*}}\left\|u_{n}-u\right\|_{Y} \leq \widehat{C}\left\|u_{n}-u\right\|_{Y} \rightarrow 0, \quad n \rightarrow \infty .
$$

Thus we arrive at the conclusion that

$$
\left\langle\Psi^{\prime}\left(u_{n}\right), u_{n}-u\right\rangle \rightarrow 0, \quad \text { as } n \rightarrow \infty .
$$

Since $\Psi^{\prime}$ enjoys the $\left(\mathrm{S}^{+}\right)$property, we have that $u_{n} \rightarrow u$ strongly in $X, n \rightarrow \infty$. Therefore $\Phi$ satisfies the Palais-Smale condition.

End of The Proof of Theorem 1.2. We verify the assumptions of Theorem 2.5. By Lemmas 3.1 and 3.2, $\Phi$ satisfies the Palais-Smale condition and is bounded from below. By $\left(f_{1}\right)$ and $\left(f_{4}\right), \Phi$ is even and $\Phi(0)=0$. Now we verify that $\Phi$ satisfies $(2.1)$.

For any $k \in \mathbb{N}$, we choose $k$ independent smooth functions $\phi_{i} \in C_{0, r}^{\infty}\left(\mathbb{R}^{N}\right)$ for $i=1, \ldots, k$ and define $X_{k}=\operatorname{span}\left\{\phi_{1}, \ldots \phi_{k}\right\}$. Then $X_{k} \subset W_{r}^{1, p}\left(\mathbb{R}^{N} ; V\right) \subset$ $L^{1}\left(\mathbb{R}^{N} ; Q\right)$ and $\operatorname{dim} X_{k}=k$. By $\left(\mathrm{f}_{3}\right)$ we have

$$
\Phi(u) \leq \frac{1}{p}\|u\|_{W_{r}^{1, p}\left(\mathbb{R}^{N} ; V\right)}^{p}-C\|u\|_{L^{1}\left(\mathbb{R}^{N} ; Q\right)} .
$$

Since all norms on $X_{k}$ are equivalent and $p>1$, we get that for $\rho_{k}>0$ small enough,

$$
\sup _{u \in X_{k} \cap S_{\rho_{k}}} \Phi(u)<0 .
$$

With all conditions of Theorem 2.5 being verified, we get the conclusion that $\Phi$ has a sequence of critical values $c_{k}<0$ satisfying $c_{k} \rightarrow 0$ as $k \rightarrow \infty$. The proof of Theorem 1.2 is complete.

We finish the paper with some remarks and with some discussions related to other topics. 
REMARK 3.3. We first give a remark on the relations between the critical points of $\Phi$ and the solutions of $(\mathrm{P})$. If $u_{0} \in W_{r}^{1, p}\left(\mathbb{R}^{N}, V\right)$ is a critical point of $\Phi$, then $u_{0}$ solves $(\mathrm{P})$ in the sense (see [2]) that

$$
\left\{\begin{aligned}
-\operatorname{div}\left(\left|\nabla u_{0}\right|^{p-2} \nabla u_{0}\right)+V(|x|)\left|u_{0}\right|^{p-2} u_{0} & \\
& \text { in }\left[Q(|x|) \underline{f}\left(u_{0}(x)\right), Q(|x|) \bar{f}\left(u_{0}(x)\right], \quad x \in \mathbb{R}^{N},\right. \\
u_{0}(x) \rightarrow 0, \quad|x| \rightarrow \infty &
\end{aligned}\right.
$$

where

$$
\underline{f}\left(u_{0}\right):=\liminf _{u \rightarrow u_{0}} f(u), \quad \bar{f}\left(u_{0}\right):=\limsup _{u \rightarrow u_{0}} f(u) .
$$

In general, such a function $u_{0}$ may not be a solution of (P). A question arising here that under what situations in which a solution of $(\widetilde{\mathrm{P}})$ becomes a solution of $(\mathrm{P})$. There was a positive answer in [3], [4] for the semlinear elliptic boundary value problems on bounded smooth domain of $\mathbb{R}^{N}$ with discontinuous nonlinearity. We refer the reader to [3], [4] for details. In the future we will investigate this question for the weighted quasilinear problems on the whole space $\mathbb{R}^{N}$.

Remark 3.4. We look at a special case of Theorem 1.2 as an example. Since the function $f(u)=\operatorname{sgn} u$ satisfies the assumptions $\left(\mathrm{f}_{1}\right)-\left(\mathrm{f}_{4}\right)$, by Theorem 2.5 , the functional

$$
\Phi(u)=\frac{1}{p} \int_{\mathbb{R}^{N}}|\nabla u|^{p}+V(|x|)|u|^{p} d x-\int_{\mathbb{R}^{N}} Q(|x|)|u| d x, \quad u \in W_{r}^{1, p}\left(\mathbb{R}^{N} ; V\right)
$$

has a sequence of critical points $\left\{u_{k}\right\}$ in $W_{r}^{1, p}\left(\mathbb{R}^{N} ; V\right)$ with $\Phi\left(u_{k}\right):=c_{k}<0$, $\Phi\left(u_{k}\right) \rightarrow 0$ as $k \rightarrow \infty$, and the quasilinear problem

$$
\left\{\begin{array}{l}
-\operatorname{div}\left(|\nabla u|^{p-2} \nabla u\right)+V(|x|)|u|^{p-2} u \in[-Q(|x|), Q(|x|)], \quad x \in \mathbb{R}^{N}, \\
u(x) \rightarrow 0, \quad|x| \rightarrow \infty
\end{array}\right.
$$

possesses infinitely many pairs of solutions in $W_{r}^{1, p}\left(\mathbb{R}^{N} ; V\right)$ with energies go to zero.

REMARK 3.5. Theorem 2.5 can be applied to the semilinear elliptic boundary value problem

$$
\begin{cases}-\Delta u=\operatorname{sgn} u, & x \in \Omega, \\ u(x)=0, & x \in \partial \Omega,\end{cases}
$$

where $\Omega$ is a bounded smooth domain of $\mathbb{R}^{N}$. Since the function $\operatorname{sgn} u$ satisfies the condition (C) (see p. 126 in [4]), $\operatorname{sgn} 0=0$, and the optimal for $(-\Delta, \operatorname{sgn})$ is still the function sgn (see [3], [4]), by Theorem 2.2 in [4], the solutions of the problem $(\overline{\mathrm{P}})$ correspond to the critical points of the functional $\bar{\Phi}$ associated to $(\overline{\mathrm{P}})$. By Theorem 2.5 and the elliptic regularity, the functional $\bar{\Phi}$ possesses infinitely many pairs of critical points in $H_{0}^{1}(\Omega)$ with their negative critical values go to 
zero and then their $H_{0}^{1}$-norm go to zero. This multiplicity result for $(\overline{\mathrm{P}})$ is new. This result should be compared to the result at the end of [2].

Acknowledgements. The authors are grateful to the anonymous referees for their helpful comments and suggestions.

\section{REFERENCES}

[1] R. Adams, Sobolev spaces, Academic Press, Amsterdam, 1975.

[2] K.C. ChAng, Variational methods for non-differentiable functionals and their applications to partial differential equations, J. Math. Anal. Appl. 80 (1981), 102-129.

[3] - On the multiple solutions of the elliptic differential equations with discontiunous nonlinear terms, Sci. Sinica 21 (1978), 139-158.

[4] The obstacle problem and partial differential equations with discontinuous nonlinearities, Commun. Pure Appl. Math. 33 (1980), 117-146.

[5] F.H. Clarck, A new approach to Lagrange multipliers, Math. Oper. Res. 1 (1976), 165-174.

[6] J.N. Corvellec, Nontrivial Solutions of quasilinear equations via nonsmooth Morse theory, J. Differential Equations 136 (1997), 268-293.

[7] J.N. Corvellec, V.V. Motreanu and C. Saccon, Doubly resonant semilinear elliptic problems via nonsmooth critical point theory, J. Differential Equations 248 (2010), 20642091.

[8] P. De NÁPoli And M.C. Mariani, Mountain pass solutions to equations of p-Laplacian type, Nonlinear Anal. 54 (2003), 1205-1219.

[9] J.Q. LIU AND Y.X. GUO, Critical point theory for nonsmooth functionals, Nonlinear Anal. 66 (2007), 2731-2741.

[10] P.H. Rabinowitz, Minimax methods in critical point theory with applications to differential equations, CBMS Regional Conf. Ser. in Math., vol. 65, Amer. Math. Soc., Providence, RI, 1986.

[11] W.A. Strauss, Existence of solitary waves in higher dimensions, Comm. Math. Phys. 55 (1977), 149-162.

[12] J.B. Su, Quasilinear elliptic Equations on $\mathbb{R}^{N}$ with singular and bounded nonlinearity, Z. Angew. Math. Phys. 63, 51-62; erratum: (2012), 63-64.

[13] J.B. Su AND R.S. TiAn, Weighted Sobolev type embeddings and coercive quasilinear elliptic equations on $\mathbb{R}^{N}$, Proc. Amer. Math. Soc. 140 (2012), 891-903.

[14] J.B. Su, Z.Q. WANG AND M. WILlEM, Nonlinear Schrödinger equations with unbounded and decaying radial potentials, Commun. Contemp. Math. 9 (2007), 571-583.

[15] Weighted Sobolev embedding with unbounded and decaying radial potentials, J. Differential Equations 238 (2007), 201-219.

[16] Z.Q. WANG, Nonlinear boundary value problems with concave nonlinearities near the origin, Nonlinear Differential Equations Appl. 8 (2001), 15-33.

[17] M. Willem, Minimax Theorems, Birkhäuser, Boston, 1996.

Anran Li, Hongrui Cai and Jiabao Su

School of Mathematical Sciences

Capital Normal University

Beijing 100048, P.R. CHINA

E-mail address: anran0200@163.com

TMNA : Volume $43-2014-\mathrm{N}^{\circ} 2$ 